\title{
Out of Center Sleep Testing in Ostensibly Healthy Middle Aged to Older Adults
}

\author{
Stuart F. Quan, M.D., 1,2 \\ Brandon J. Lockyer ${ }^{1}$ \\ Salma Batool-Anwar, M.D., M.P.H. ${ }^{1}$ \\ Daniel Aeschbach, Ph.D. ${ }^{1,3}$
}

${ }^{1}$ Division of Sleep and Circadian Disorders, Department of Medicine and Department of

Neurology; Brigham and Women's Hospital and Division of Sleep Medicine, Harvard Medical School, Boston, MA

${ }^{2}$ Asthma and Airways Research Center, University of Arizona College of Medicine, Tucson, AZ

${ }^{3}$ Department of Sleep and Human Factors Research; Institute of Aerospace Medicine; German Aerospace Center; Cologne, 51147; Germany

\begin{abstract}
Background: Out of Center Sleep Testing (OCST) is used increasingly to diagnose obstructive sleep apnea (OSA). However, there are few data using OCST that quantify the amount of intrinsic apneic and hypopneic events among asymptomatic healthy persons, especially those who are elderly. This analysis reports the results of OCST in a small group of ostensibly healthy asymptomatic individuals.

Methods: The study population was comprised of ostensibly healthy middle-aged to elderly volunteers for studies of circadian physiology. Before undergoing an OCST, they were found to be free of any chronic medical or psychiatry condition by history, physical and psychologic examination and by a variety of questionnaires and laboratory tests. Results: There were 24 subjects ranging in age from 55-70 years who had an OCST performed. Repeat studies were required in only 3 subjects. Over half the study population was over the age of 60 years $(54.2 \%$ vs $45.8 \%)$; the majority were men $(70.8 \%)$. The mean apnea hypopnea index $(\mathrm{AHI})$ was 9.2 /hour with no difference between younger and older subjects. However, 11 had an $\mathrm{AHI} \geq 5$ /hour. Five had an $\mathrm{AHI} \geq 15$ /hour and 2 had an $\mathrm{AHI} \geq 40$ /hour. Those with an $\mathrm{AHI}<15$ /hour had a mean AHI of 4.4 /hour (95\% Cl:2.8-6.0/hour).

Conclusions: Although OCST has a low failure rate, there is a high prevalence of intrinsic obstructive apnea and hypopnea in ostensibly healthy asymptomatic persons.
\end{abstract}

\section{Introduction}

Out of center sleep testing (OCST) is increasingly used instead of laboratory polysomnography (PSG) for the identification of persons with obstructive sleep apnea (OSA) (1). There have been a number of studies validating the use of OCST for this purpose (1). Consequently, large research studies now are using OCST to identify subjects with OSA for clinical trials or observational studies (2-4). Out of center sleep testing also may have a role in identifying the presence of unrecognized OSA in studies 
of asymptomatic normal individuals. Studies reporting on the frequency of apneic and hypopneic events in healthy individuals using current PSG monitoring techniques $(5,6)$ found surprisingly high prevalence rates of OSA. Similar reports using OCST also noted that OSA was common in asymptomatic volunteers, but many of these subjects still had chronic medical conditions $(7,8)$. Thus, there is little information on the prevalence of OSA using OCST, especially in those who are older without any co-morbidities.

In this report, we describe the results of OCST in a small group of middle-aged to older adults who were screened intensively to exclude the presence of chronic sleep and medical conditions. We hypothesized that in this group of ostensibly healthy adults the prevalence of OSA would be less than previously observed.

\section{Methods}

Potential subjects between 55 and 70 years of age were identified through public advertisements for volunteers to participate in a circadian physiology research study. The study was approved by the Partners Health Care Human Research Committee, and subjects gave written informed consent prior to their participation. Initially, 5,225 individuals were screened by telephone for medical and psychiatric exclusion criteria. Those who passed $(n=90)$ were invited for an interview and more intensive screening which included a medical history, the Pittsburgh Sleep Quality Index (PSQI) (9), Epworth Sleepiness Scale (ESS) (10) the Berlin Questionnaire (11), blood and urine tests, an electrocardiogram, and a complete physical examination by a physician. In addition, a structured psychologic examination was conducted by a clinical psychologist along with completion of several psychological screening tests including the Geriatric Depression Scale (12), Mattis Dementia Rating Scale (13) and the Mini Mental Status Examination (14). Potential subjects also could not have worked at night for the previous 3 years or recently traveled across time zones. Those who were determined to be free from any acute or chronic medical or psychiatric condition including but not limited to obesity (body mass index, $\mathrm{BMI}<30 \mathrm{~kg} / \mathrm{m}^{2}$ ), medication use, hypertension, cardiovascular or pulmonary disease, neoplasia, and disorders of the gastrointestinal, renal, endocrine, metabolic or neurologic systems were asked to undergo an OCST.

The OCST was performed using the Embletta Gold (Embla Systems, Broomfield, CO), a Type III OCST device. The testing montage included nasal pressure, pulse oximetry, bilateral leg electromyography, and chest and abdominal inductance plethysmography. Subjects were instructed to sleep at their habitual hours during OCST. Procedure for determining lights out and lights on, sleep onset and sleep offset, and wake periods during OCST in the sleep, aging and circadian rhythm disorders is given in Appendix 1. Estimated total sleep time was ascertained by the scoring technologist on the basis of changes in the recorded signals indicative of sleep onset or offset and a questionnaire administered to the subject on the morning after the study (see online supplement for the protocol). Studies were scored for apneas, hypopneas and periodic limb movements according to the following criteria. An apnea was defined as $>90 \%$ amplitude decrease from baseline of the nasal pressure signal lasting $\geq 10 \mathrm{~s}$. Hypopneas were scored if an event was at least $10 \mathrm{~s}$ in duration and if there was a clear amplitude reduction of the 
nasal pressure signal that was associated with an oxygen desaturation $\geq 4 \%$. Obstructive or central apneas were identified by the presence or absence of respiratory effort, respectively. The apnea hypopnea index (AHI) was calculated as the sum of all apneas and hypopneas divided by the estimated total sleep time. Periodic limb movements were identified using American Academy of Sleep Medicine criteria (15). The periodic limb movement index (PLMI) was computed as the sum of all periodic limb movements divided by the estimated total sleep time. All OCST recordings were scored by a registered polysomnographic technologist and reviewed by a board-certified sleep physician.

Six subjects who had successfully completed the circadian physiology protocol were invited again to enroll in a related protocol approximately 1-3 years later. They repeated the aforementioned screening procedures including a second OCST.

Paired and unpaired Student's t-tests were used to compare means. Comparisons between proportions were performed using $\chi^{2}$. Data are expressed as means \pm SD or number and \% of cases. Data were analyzed using IBM SPSS Statistics V24 (Armonk, NY).

\section{Results}

As shown in Table 1, there were 24 subjects who successfully underwent a screening OCST.

\begin{tabular}{|c|c|c|c|c|}
\hline & All Subjects & $50-59$ years & $>60$ year & $\begin{array}{c}p \\
\text { value }\end{array}$ \\
\hline $\mathbf{N}$ & 24 & 11 & 13 & \\
\hline Age (years) & $60.3 \pm 4.5$ & $56.6 \pm 1.3$ & $63.5 \pm 3.5$ & $<0.001$ \\
\hline Gender (M/F) & $17 / 7$ & $7 / 4$ & $10 / 3$ & 0.395 \\
\hline Race $(w / 0) t$ & $18 / 6$ & $6 / 5$ & $12 / 1$ & 0.182 \\
\hline Body Mass Index (kg/m2) & $25.6 \pm 3.4$ & $27.1 \pm 2.7$ & $24.2 \pm 3.5$ & 0.036 \\
\hline Berlin Score $\mp$ & 7 & 4 & 3 & 0.570 \\
\hline Epworth Sleepiness Scale & $3.3 \pm 2.1$ & $3.7 \pm 2.5$ & $2.9 \pm 1.6$ & 0.362 \\
\hline Pittsburgh Sleep Quality Index & $2.4 \pm 1.6$ & $2.0 \pm 1.3$ & $2.8 \pm 1.9$ & 0.234 \\
\hline Estimated Total Sleep Time (hours) & $8.5 \pm 1.7$ & $9.2 \pm 0.6$ & $7.9 \pm 2.1$ & 0.059 \\
\hline Apnea Hypopnea Index (\#/h) & $9.2 \pm 11.4$ & $8.9 \pm 11.2$ & $9.4 \pm 11.9$ & 0.903 \\
\hline$\#$ of Subjects with AHI >5 hour & 11 & 5 & 6 & 0.647 \\
\hline$\#$ of Subjects with AHI >15 hour & 5 & 2 & 3 & 0.585 \\
\hline SaO2 Average $(\%)$ & $95.5 \pm 1.5$ & $94.9 \pm 1.4$ & $96.1 \pm 1.4$ & 0.061 \\
\hline Time Spent with SaO2<90\% (min) & $0.27 \pm 0.62$ & $0.48 \pm .88$ & $0.10 \pm 0.17$ & 0.140 \\
\hline Periodic Limb Movement Index ( $\# / \mathrm{h})$ & $3.9 \pm 4.6$ & $2.3 \pm 2.8$ & $5.2 \pm 5.5$ & 0.137 \\
\hline \multicolumn{5}{|l|}{${ }^{*}$ Data shown as mean \pm SD } \\
\hline \multicolumn{5}{|l|}{ tW=Non-Hispanic White; $\mathbf{O}=0$ ther } \\
\hline$\ddagger$ \#erlin Score=\# Subjects with 1 categ & & & & \\
\hline
\end{tabular}


Their ages ranged from 55 to 70 years. Over half the study population was over the age of 60 years [13/24 (54.2\%) vs 11/24 (45.8\%)], and the majority were men (70.8\%). In 3 subjects, the initial OCST provided insufficient data and a repeat study was required. The mean $\mathrm{AHI}$ was 9.2 /hour overall and there were no differences between younger and older subjects. Notably, 11 subjects $(46 \%)$ had an AHI greater than 5 /hour. Five (21\%) had an $\mathrm{AHI}$ greater than 15 /hour and 2 subjects had an $\mathrm{AHI}$ greater than 40 /hour. Excluding subjects with an AHI $\geq 15$ /hour yielded a mean AHI of 4.4 (95\% Cl: 2.86.0) /hour. Younger subjects had a higher BMl ( $27.1 \pm 2.7 \mathrm{vs} .24 .2 \pm 3.5 \mathrm{~kg} / \mathrm{m}^{2}$, $p=0.036)$, tended to have a longer estimated total sleep time $(9.2 \pm 0.6$ vs. $7.9 \pm 2.1$ hours, $p=0.059)$, and a slightly lower average nocturnal oxygen saturation (94.9 \pm 1.4 vs. $96.1 \pm 1.4 \%, p=.061$ ). No other differences were observed between young and older subjects.

In Table 2 are shown the sleep and anthropometric findings for the 6 subjects who had repeat testing.

\begin{tabular}{|c|c|c|}
\hline & 1st Evaluation & 2nd Evaluation \\
\hline Epworth Sleepiness Scale & $3.2 \pm 2.9$ & $3.2 \pm 2.3$ \\
\hline Pittsburgh Sleep Quality Index & $3.2 \pm 1.7$ & $2.5 \pm 1.4$ \\
\hline Estimated Total Sleep Time (hours) & $9.4 \pm 0.5$ & $8.5 \pm 1.6$ \\
\hline Apnea Hypopnea Index (\#/h) & $5.2 \pm 4.1$ & $6.2 \pm 2.3$ \\
\hline Periodic Limb Movement Index (\#/h) & $1.7 \pm 1.8$ & $3.3 \pm 4.0$ \\
\hline Body Mass Index $(\mathrm{kg} / \mathrm{m} 2) \neq$ & $25.8 \pm 4.8$ & $26.4 \pm 5.0$ \\
\hline \multicolumn{3}{|l|}{$* \mathrm{~N}=6$} \\
\hline \multicolumn{3}{|l|}{ tData shown as meantSD } \\
\hline \multicolumn{3}{|l|}{$\ddagger \mathrm{p}=0.027$} \\
\hline IDays between evaluation: $672 \pm 227$ ( & 30) & \\
\hline
\end{tabular}

The interval between tests ranged from 382 to 930 days (mean: $672 \pm 227$ days). Although there was a slight increase in BMI over this interval, no changes were noted in their sleep quality, AHI or PLMI.

\section{Discussion}

In this study, we found that it was feasible to screen an ostensibly healthy asymptomatic group of middle to older aged adults for the presence of OSA using a Level III OCST device. The results of the OCST were replicable over an interval of several years and there were few technical failures. Furthermore, evidence of OSA was observed in a 
substantial proportion of these individuals underscoring that the diagnosis of OSA remains unrecognized in many elderly persons.

Over the past 20 years, there have been numerous clinical trials and cohort studies that have recorded data related to the presence of OSA $(2-4,8,16,17)$. Most have used PSG recorded in the laboratory or at home (16-18). However, the use of PSG is logistically complex and expensive. Additionally, the equipment required for data acquisition may artifactually disrupt the subject's sleep. In contrast, OCST is less intrusive and expensive, but actual sleep is not recorded. However, to our knowledge there have not been previous studies that have determined the normative values for the $\mathrm{AHI}$ using OCST in a group of ostensibly healthy middle-aged to older adults. In this small cohort, the mean AHI was 9.2 /hour. According to the $3^{\text {rd }}$ International Classification of Sleep Disorders (19), an AHI $\geq 15$ /hour is diagnostic of OSA even in the absence of symptoms or associated medical conditions. Therefore, after excluding those who met criteria for at least moderate OSA (i.e., AHI $\geq 15$ hour), our findings indicate an average value of 4.4 /hour in those individuals with a "normal" $\mathrm{AHI}$ using OCST. In contrast, a previous study of healthy volunteers for circadian physiology research using similar inclusion and exclusion criteria found the overall mean $\mathrm{AHI}$ of all subjects using PSG was substantially higher; hypopneas were identified if reductions in airflow were associated with a minimum 3\% oxygen desaturation or a cortical arousal (6). However, a more recent study also using PSG found AHI values more similar to our observations (5). Although it would be expected that OCST may underreport the presence of hypopneas, the explanation for the differences between the two PSG studies is unclear given that the recording montages and respiratory scoring algorithms appeared to be similar.

A striking finding from this study is the high prevalence of OSA among these carefully screened, ostensibly healthy volunteers who had no evidence of a sleep disorder based on their Berlin Questionnaire, PSQI and ESS. The AHI of 2 subjects documented the presence of severe OSA which is usually an indication for treatment. The absence of hypersomnia in a substantial proportion of persons with PSG evidence of OSA has been clearly documented (20). Our data extend these observations by demonstrating the existence of severe OSA in ostensibly healthy middle-aged to older adults. Although some data suggest that such individuals are not at risk for cardiovascular disease or other sequelae of OSA (8), whether this prognosis is correct is still not settled.

Some, but not all epidemiologic data suggest that OSA slowly worsens with age. In contrast, we observed no progression of OSA over an interval of approximately 1-2.5 years $(8,21)$. However, it is likely that our follow up interval was insufficient to detect a change given the small number of subjects.

In conclusion, use of OCST to screen for OSA is feasible in research studies of normal individuals. Importantly, a relatively high proportion of even ostensibly healthy individuals can be expected to show evidence of OSA. 


\section{Acknowledgements}

This study was supported by P01 AG009975 from the National Institute of Aging. We would like to express our gratitude to Alec Rader and Jacob Medina for their help with subject recruitment and Stephanie Marvin for scoring support.

\section{References}

1. Kapur VK, Auckley DH, Chowdhuri S, et al. Clinical Practice Guideline for Diagnostic Testing for Adult Obstructive Sleep Apnea: An American Academy of Sleep Medicine Clinical Practice Guideline. J Clin Sleep Med. 2017;13(3):479-504. [CrossRef] [PubMed]

2. Gottlieb DJ, Punjabi NM, Mehra R, et al. CPAP versus oxygen in obstructive sleep apnea. N Engl J Med. 2014;370(24):2276-85. [CrossRef] [PubMed]

3. Zhao YY, Wang R, Gleason KJ, et al. Effect of Continuous Positive Airway Pressure Treatment on Health-Related Quality of Life and Sleepiness in High Cardiovascular Risk Individuals with Sleep Apnea: Best Apnea Interventions for Research (BestAIR) Trial. Sleep. 2017;40(4):10.1093/sleep/zsx040. [CrossRef] [PubMed]

4. Redline S, Sotres-Alvarez D, Loredo J, et al. Sleep-disordered breathing in Hispanic/Latino individuals of diverse backgrounds. The Hispanic Community Health Study/Study of Latinos. Am J Respir Crit Care Med. 2014;189(3):335-44. [CrossRef] [PubMed]

5. Mitterling T, Hogl B, Schonwald SV, et al. Sleep and Respiration in 100 Healthy Caucasian Sleepers--A Polysomnographic Study According to American Academy of Sleep Medicine Standards. Sleep. 2015;38(6):867-75. [CrossRef] [PubMed]

6. Pavlova MK, Duffy JF, Shea SA. Polysomnographic respiratory abnormalities in asymptomatic individuals. Sleep. 2008;31(2):241-8. [CrossRef] [PubMed]

7. Sforza E, Pichot V, Martin MS, Barthelemy JC, Roche F. Prevalence and determinants of subjective sleepiness in healthy elderly with unrecognized obstructive sleep apnea. Sleep Med. 2015;16(8):981-6.[CrossRef] [PubMed]

8. Sforza E, Hupin D, Pichot V, Barthelemy JC, Roche F. A 7-year follow-up study of obstructive sleep apnoea in healthy elderly: The PROOF cohort study. Respirology. 2017;22(5):1007-14. [CrossRef] [PubMed]

9. Buysse DJ, Reynolds CF,3rd, Monk TH, Berman SR, Kupfer DJ. The Pittsburgh Sleep Quality Index: a new instrument for psychiatric practice and research.

Psychiatry Res. 1989;28(2):193-213. [CrossRef] [PubMed]

10. Johns MW. A new method for measuring daytime sleepiness: the Epworth sleepiness scale. Sleep. 1991;14(6):540-5. [CrossRef] [PubMed]

11. Netzer NC, Stoohs RA, Netzer CM, Clark K, Strohl KP. Using the Berlin Questionnaire to identify patients at risk for the sleep apnea syndrome. Ann Intern Med. 1999;131(7):485-91.[CrossRef] [PubMed]

12. Yesavage JA, Brink TL, Rose TL, et al. Development and validation of a geriatric depression screening scale: a preliminary report. J Psychiatr Res. 1982;17(1):37-49. [CrossRef] [PubMed] 
13. Mattis S. Mental status examination for organic mental syndrome in the elderly patient. In: Geriatric psychiatry. Bellak L and Karasu TE, eds. New York: Grune and Stratton, 1976; 77-121

14. Pangman VC, Sloan J, Guse L. An examination of psychometric properties of the mini-mental state examination and the standardized mini-mental state examination: implications for clinical practice. Appl Nurs Res. 2000;13(4):209-213. [CrossRef] [PubMed]

15. Iber C, Ancoli-Israel S, Chesson A, Quan SF. The AASM Manual for the Scoring of Sleep and Associated Events: Rules, Terminology and Technical Specifications. 1st ed. Westchester, IL: American Academy of Sleep Medicine, 2007; 57.

16. Quan SF, Howard BV, Iber C, et al. The Sleep Heart Health Study: design, rationale, and methods. Sleep. 1997;20(12):1077-85. [PubMed]

17. Kushida CA, Nichols DA, Quan SF, et al. The Apnea Positive Pressure Long-term Efficacy Study (APPLES): rationale, design, methods, and procedures. J Clin Sleep Med. 2006;2(3):288-300. [PubMed]

18. Chen X, Wang R, Zee $P$, et al. Racial/Ethnic Differences in Sleep Disturbances: The Multi-Ethnic Study of Atherosclerosis (MESA). Sleep. 2015;38(6):877-88. [CrossRef] [PubMed]

19. Sateia MJ. International Classification of Sleep Disorders. 3rd ed. Darien, IL: American Academy of Sleep Medicine, 2014.

20. He K, Kapur VK. Sleep-Disordered Breathing and Excessive Daytime Sleepiness. Sleep Med Clin. 2017;12(3):369-82.[CrossRef] [PubMed]

21. Redline S, Schluchter MD, Larkin EK, Tishler PV. Predictors of longitudinal change in sleep-disordered breathing in a nonclinic population. Sleep. 2003;26(6):703-9. [CrossRef] [PubMed] 